\title{
AN INFRARED SINGULARITY IN THE DAMPING RATE FOR LONGITUDINAL GLUONS IN HOT QCD
}

\author{
A. Abada and O. Azi \\ Département de Physique, École Normale Supérieure \\ BP 92 Vieux Kouba, 16050 Alger, Algeria \\ enskppps@ist.cerist.dz
}

(March 22, 2018)

\begin{abstract}
We calculate $\gamma_{l}(0)$, the damping rate for longitudinal on-shell gluons with zero momentum in hot QCD using the hard-thermal-loop (htl) scheme. We find it to be divergent in the infrared, which means that in this scheme $\gamma_{l}(0)$ is different from $\gamma_{t}(0)$, the corresponding damping rate for transverse gluons which is known to be finite. This result suggests that the htl scheme is infrared sensitive and thus may need to be improved upon in this sector. We discuss this issue after we present our calculation.
\end{abstract}

pacs: $11.10 . \mathrm{Wx}$ 12.38.-t 12.38.Bx 12.38.Mh

keywords: hard thermal loops. soft gluon damping.

ENSK-TP-10

Besides their importance regarding the stability of the quark-gluon plasma, gluon damping rates have been crucial in better understanding QCD at high temperature $T$. In early works, it has been noticed that in this regime, the determination of the dispersion laws for quarks and gluons beyond lowest order using standard perturbation theory is gaugedependent [1]. This problem has been emphasized in further works in which the gluon damping rates have been calculated to one-loop order in various gauges and schemes and different results have been obtained [2]. It was then realized that the problem was related to the way the expansion in powers of $g$, the perturbative QCD coupling constant, was performed: at high $T$ (the hard scale), when the external momenta are soft, i.e., of magnitude $g T$, the standard loop expansion is not anymore an expansion in powers of $g$ [3]. It was subsequently developed an effective perturbative expansion in the framework of a resummation scheme of the so-called hard thermal loops (htl) [4]. Using this scheme, the transverse-gluon damping rate $\gamma_{t}(0)$ with zero momentum was shown to be Coulomb-and-covariant-gaugeinvariant and determined in the strict Coulomb gauge to be finite and positive [5]. Later, a 
generating-functional formalism in the htl approximation was developed [6] and a relation to the eikonal of a Chern-Simons gauge theory was made [7]. From there a hydrodynamic approach showed that the htl approximation was essentially 'classical' [8].

However, the htl resummation scheme may be not 'complete' yet in describing the whole picture of QCD at high temperature. Indeed, it discusses only the two scales $T$ (hard) and $g T$ (soft) whereas with $g$ and $T$, one has (at least) a hierarchy of scales $g^{n} T, n$ an integer, positive if we believe that $T$ is the highest scale in the problem. It may be unnecessary to reorganize perturbation theory taking into account this general structure of scales, but there are indications that at least the next smaller one $g^{2} T$ may be important. Indeed, it is true that in the htl scheme, both on-shell longitudinal and transverse gluons acquire a thermal mass $m_{g}$ of order $g T$, but static magnetic fields are not screened yet at this scale [9]; they are expected to get so at the hence-called 'magnetic scale' $g^{2} T$. Furthermore, the gluon damping rates $\gamma(p)$ are to lowest order of magnitude $g^{2} T$ and it has been noticed that these damping rates, when calculated in the htl-resummation scheme at soft but nonzero momenta $p$, exhibit a $\ln g$ behavior accompanied with coefficients [10]. This logarithmic behavior is not present in the expression of $\gamma_{t}(0)$, the limit $p \rightarrow 0$ of $\gamma_{t}(p)$, and no explanation has been provided yet for this discrepancy. These remarks tend to indicate therefore that the htl scheme may be sensitive in the 'infrared'.

We think a more direct indication of the infrared sensitivity of the htl-resummation scheme is the observation we made in [11] that, when calculated solely in this scheme, the longitudinal-gluon damping rate at zero momentum $\gamma_{l}(0)$ may be potentially infrareddivergent. Indeed, we have determined in that work the analytic expression to leading order of $\gamma_{l}(0)$. We have obtained for it the following form:

$$
\gamma_{l}(0)=\frac{g^{2} N_{c} T}{24 \pi}\left[a_{l 0}^{(1)}+a_{l 0}^{(2)}\right]
$$

$N_{c}$ is the number of colors. $a_{l 0}^{(1)}$ is given in eq (3) below; it is a finite number found in [5] to be equal to 6.63538 and is such that the damping rate $\gamma_{t}(0)$ for transverse gluons with zero momentum is equal to $\frac{g^{2} N_{c} T}{24 \pi} a_{l 0}^{(1)}$, see [5]. The other contribution $a_{l 0}^{(2)}$ is given in eq (4) and it is this part of $\gamma_{l}(0)$ we remarked it contains terms which are divergent in the infrared [11].

From a physical standpoint and independently of any calculation scheme, there is no difference at zero momentum between longitudinal and transverse gluons, and hence one expects any consistent scheme to yield the same result for both damping rates [5]. In virtue of this physical argument, once $\gamma_{t}(0)$ was found in the htl scheme to be finite and positive, $\gamma_{l}(0)$ was thought to be so and to the best of our knowledge, no explicit calculation of the latter in the htl scheme has been reported prior to the one in [11]. With this in mind and expecting the htl-resummation scheme to be 'complete', we argued in 11 that the terms contributing to $a_{l 0}^{(2)}$, in particular the infrared-divergent ones, when put together, may cancel 
one another. We show in this letter that this is not the case: when all the expressions are treated with care, not only we find the part $a_{l 0}^{(2)}$ nonvanishing but the infrared-divergent piece survives too, see eq (23). In the sequel, we present first our calculation and we discuss the result afterwards.

The longitudinal gluon damping rate in the strict Coulomb gauge is obtained to lowest order by the relation:

$$
\gamma_{l}(p)=\left.\frac{\operatorname{Im}^{*} \Pi_{l}(-i \omega, p)}{\frac{\partial}{\partial \omega} \delta \Pi_{l}(-i \omega, p)}\right|_{\omega=\omega_{l}(p)+i 0^{+}}
$$

where $\delta \Pi_{l}\left({ }^{*} \Pi_{l}\right)$ is the longitudinal-gluon htl (effective) self-energy. $\omega_{l}(p)$ is its on-shell lowest-order energy given for soft momenta in units of $m_{g}$ by eq (8) below. We follow closely the notation of 11] to which this work is a follow-up. Since the denominator in (2) is given in units of $m_{g}$ by $\partial /\left.\partial \omega \delta \Pi(-i \omega, p)\right|_{\omega=\omega_{l}(p)}=-2 p^{2}+\ldots$, to get $\gamma_{l}(p=0)$, we have to expand the imaginary part of the effective self-energy to order $p^{2}$. This calculation is reported in [11 and we obtain the result (11) where the expressions of $a_{l 0}^{(1)}$ and $a_{l 0}^{(2)}$ are the following:

$$
\begin{gathered}
a_{l 0}^{(1)}=9 \int_{0}^{+\infty} d k \int_{-\infty}^{+\infty} \frac{d \omega_{1}}{\omega_{1}} \int_{-\infty}^{+\infty} \frac{d \omega_{2}}{\omega_{2}} \delta\left(1-\omega_{1}-\omega_{2}\right)\left[k^{4} \rho_{l 1} \rho_{l 2}-k^{2}\left(k^{2}-\omega_{1}^{2}\right)^{2}\right. \\
\left.\times \rho_{t 1} \rho_{l 2}+2\left(k^{2}+\omega_{1} \omega_{2}\right)^{2} \rho_{t 1} \rho_{t 2}+\frac{\omega_{1}}{6 k^{3}}\left(k^{2}-\omega_{1}^{2}\right)^{2} \Theta_{1} \rho_{t 2}\right]
\end{gathered}
$$

and:

$$
\begin{aligned}
a_{l 0}^{(2)}= & \int_{0}^{+\infty} d k \int_{-\infty}^{+\infty} \frac{d \omega_{1}}{\omega_{1}} \int_{-\infty}^{+\infty} \frac{d \omega_{2}}{\omega_{2}} \delta\left(1-\omega_{1}-\omega_{2}\right)\left[\frac{\omega_{1}}{3}\left(1-2 \omega_{1}\right) \Theta_{1} \partial_{k} \rho_{l 2}+\frac{\omega_{1} k}{6} \Theta_{1} \partial_{k}^{2} \rho_{l 2}\right. \\
& +\frac{\omega_{1}}{3}\left(1-\frac{\omega_{1}^{2}}{k^{2}}\right)\left(1-2 \omega_{1}\right) \partial_{k} \Theta_{1} \rho_{t 2}+\frac{\omega_{1} k}{3}\left(1-\frac{\omega_{1}^{2}}{k^{2}}\right) \partial_{k} \Theta_{1} \partial_{k} \rho_{t 2}+\frac{\omega_{1}}{6 k}\left(k^{2}-\omega_{1}^{2}\right) \partial_{k}^{2} \Theta_{1} \rho_{t 2} \\
& +\partial_{k}\left[k^{3}\left(1+\frac{k^{2}}{3}\right) \rho_{l 1} \rho_{l 2}+\frac{2 k}{9}\left(k^{2}\left(1-3 k^{2}\right)+\left(1-4 k^{2}\right) \omega_{1} \omega_{2}-\omega_{1}^{2} \omega_{2}^{2}\right) \rho_{t 1} \rho_{t 2}\right. \\
& +\frac{2 \omega_{1}^{2}}{3}\left(1+\frac{\omega_{1}}{k^{2}}-\frac{\omega_{1}^{2}}{k^{2}}\right) \Theta_{1} \rho_{t 2}+\frac{k^{2}}{9}\left(1-2 k^{2}-4\left(1-k^{2}\right) \omega_{1}+6 \omega_{1}^{2}-4 \omega_{1}^{3}\right) \rho_{t 1} \partial_{k} \rho_{t 2} \\
& \left.\left.-\frac{\omega_{1}}{6} \partial_{k}\left(\left(k-\frac{\omega_{1}^{2}}{k}\right) \Theta_{1} \rho_{t 2}\right)\right]+\frac{2}{3}\left|\omega_{1}\right|\left(2 k^{2} \delta_{1} \rho_{l 2}-k^{2} \omega_{1} \partial_{\omega_{1}^{2}} \delta_{1} \rho_{l 2}+\omega_{1} \delta_{1} \rho_{t 2}\right)\right] .
\end{aligned}
$$

The notation is as follows: $\Theta_{1} \equiv \Theta\left(k^{2}-\omega_{1}^{2}\right)$ where $\Theta$ is the step function and $\delta_{1} \equiv \delta\left(k^{2}-\omega_{1}^{2}\right)$. $\partial_{k} \equiv \partial / \partial k$ etc and $\rho_{l i}$ is a short notation for $\rho_{l}\left(\omega_{i}, k\right), i=1,2$ and the same for $\rho_{t i}$. The spectral densities $\rho_{t, l}$ are given by [5, 12,

$$
\rho_{t, l}(\omega, k)=\mathfrak{z}_{t, l}(k)\left[\delta\left(\omega-\omega_{t, l}(k)\right)-\delta\left(\omega+\omega_{t, l}(k)\right)\right]+\beta_{t, l}(\omega, k) \Theta\left(k^{2}-\omega^{2}\right),
$$

an expression in which the residues $\mathfrak{z}_{t, l}(k)$ are given by:

$$
\mathfrak{z}_{t}(k)=\left.\frac{\left(\omega^{2}-k^{2}\right)}{2\left(3 \omega^{2}-\left(\omega^{2}-k^{2}\right)^{2}\right)}\right|_{\omega=\omega_{t}(k)} ; \quad \mathfrak{z}_{l}(k)=\left.\frac{-\left(\omega^{2}-k^{2}\right)}{2 k^{2}\left(3-\omega^{2}+k^{2}\right)}\right|_{\omega=\omega_{l}(k)},
$$


and the cut functions $\beta_{t, l}(\omega, k)$ by:

$$
\begin{aligned}
& \beta_{t}(\omega, k)=\frac{3 \omega\left(k^{2}-\omega^{2}\right)}{4 k^{3}\left[\left(k^{2}-\omega^{2}+\frac{3 \omega^{2}}{2 k^{2}}\left(1+\frac{k^{2}-\omega^{2}}{2 \omega k} \ln \frac{k+\omega}{k-\omega}\right)\right)^{2}+\left(\frac{3 \pi \omega}{4 k^{3}}\left(k^{2}-\omega^{2}\right)\right)^{2}\right]} \\
& \beta_{l}(\omega, k)=\frac{-3 \omega}{2 k\left[\left(3+k^{2}-\frac{3 \omega}{2 k} \ln \frac{k+\omega}{k-\omega}\right)^{2}+\left(\frac{3 \pi \omega}{2 k}\right)^{2}\right]} .
\end{aligned}
$$

$\omega_{t, l}(p)$ are the on-shell energies of the gluon to lowest order. For soft gluons and in units of $m_{g}$, we have:

$$
\begin{aligned}
& \omega_{t}(p)=\left[1+\frac{3}{5} p^{2}-\frac{9}{35} p^{4}+\frac{704}{3000} p^{6}-\frac{91617}{336875} p^{8}+\ldots\right] \\
& \omega_{l}(p)=\left[1+\frac{3}{10} p^{2}-\frac{3}{280} p^{4}+\frac{1}{6000} p^{6}+\frac{489}{43120000} p^{8}+\ldots\right] .
\end{aligned}
$$

As we said, $a_{l 0}^{(1)}$ is a finite number found in [5] to be equal to 6.63538. We therefore have to calculate $a_{l 0}^{(2)}$. The sort of difficulties one encounters when dealing with the expressions involved in eq (4) can partly be displayed in the following simple example. Consider the integral:

$$
\begin{aligned}
I & =12 \int_{0}^{+\infty} d k \int_{-\infty}^{+\infty} \frac{d \omega_{1}}{\omega_{1}} \int_{-\infty}^{+\infty} \frac{d \omega_{2}}{\omega_{2}} \delta\left(1-\omega_{1}-\omega_{2}\right) k^{2}\left|\omega_{1}\right| \mathfrak{z}_{l}(k) \delta\left(k^{2}-\omega_{1}^{2}\right) \delta\left(\omega_{2}-\omega_{l}(k)\right) \\
& =12 \int_{0}^{+\infty} d k \int_{-\infty}^{+\infty} d \omega \frac{\mathfrak{z}_{l}(k) k^{2} \epsilon(\omega)}{1-\omega} \delta\left(k^{2}-\omega^{2}\right) \delta\left(1-\omega-\omega_{l}(k)\right),
\end{aligned}
$$

the residue piece of a term that intervenes in the expression of $a_{l 0}^{(2)}$. Here $\epsilon(\omega)$ is the sign function. We may choose to write $\delta\left(k^{2}-\omega^{2}\right)=\frac{1}{2 k} \delta(k-|\omega|)$, in which case we obtain:

$$
\begin{aligned}
I & =-6 \int_{0}^{+\infty} d k \frac{k \mathfrak{z}_{l}(k)}{\omega_{l}(k)} \delta\left(k+1-\omega_{l}(k)\right)=-\left.6 \frac{k \mathfrak{z}_{l}(k)}{\omega_{l}(k)}\left|\frac{d\left(1+k-\omega_{l}(k)\right)}{d k}\right|^{-1}\right|_{k \rightarrow 0} \\
& =\frac{3}{2 \eta}+\frac{9}{10}+O(\eta) .
\end{aligned}
$$

We have used the fact that $\omega_{l}(k) \geq 1$ for all $k$ and an expansion of $\mathfrak{z}_{l}(k)$ and $\omega_{l}(k)$ for small $k$. In the above equation, $\eta$ is an infrared cutoff; $\eta \ll 1$. Thus $I$ is divergent in the infrared. But we may also choose to write $\delta\left(k^{2}-\omega^{2}\right)=\frac{1}{2|\omega|} \delta(k-|\omega|)$, in which case we have:

$$
\begin{aligned}
I & =6 \int_{0}^{+\infty} d k \frac{k^{2} \mathfrak{z}_{l}(k)}{\omega_{l}(k)\left(1-\omega_{l}(k)\right)} \delta\left(k+1-\omega_{l}(k)\right) \\
& =\left.6 \frac{k^{2} \mathfrak{z}_{l}(k)}{\omega_{l}(k)\left(1-\omega_{l}(k)\right)}\left|\frac{d\left(1+k-\omega_{l}(k)\right)}{d k}\right|^{-1}\right|_{k \rightarrow 0}=\frac{5}{\eta^{2}}+\frac{3}{\eta}-\frac{353}{140}+O(\eta),
\end{aligned}
$$

a divergent result too, but different from that of eq (10). Which result should then one consider? The ambiguity comes from the fact that the condition $k+1-\omega_{l}(k)=0$ is 
satisfied only at $k=0$; it cannot be 'approached from above', so to speak. Thus if one chooses to regularize $I$ by defining:

$$
I_{\eta}=12 \int_{\eta>0}^{+\infty} d k \int_{-\infty}^{+\infty} \frac{d \omega_{1}}{\omega_{1}} \int_{-\infty}^{+\infty} \frac{d \omega_{2}}{\omega_{2}} \delta\left(1-\omega_{1}-\omega_{2}\right) k^{2}\left|\omega_{1}\right| \mathfrak{z}_{l}(k) \delta\left(k^{2}-\omega_{1}^{2}\right) \delta\left(\omega_{2}-\omega_{l}(k)\right),
$$

with $\eta \ll 1$ but kept different from zero until all intermediary steps are performed, for both choices of eqs (10) and (11) one obtains:

$$
I_{\eta}=0
$$

and the ambiguity is lifted. This is the regularization procedure we adopt in this work. The calculation becomes then mainly a matter of straightforwardly disentangling the infrareddivergent pieces from the finite ones. It very often necessitates an expansion for small $k$ of the residue and cut functions using their definitions given in (6) and (7) respectively and some of their derivatives. Also, care must be taken when handling the delta functions that occur, especially with their first and second derivatives. Finally, extra care must be given to the order in which the integrals over $k$ and $\omega$ are performed; it can be quite subtle in some cases.

We now give the results for the different contributions to $a_{l 0}^{(2)}$. To ease the notation, we denote in a compact way:

$$
\int \mathcal{D} \equiv 9 \int_{\eta}^{+\infty} d k \int_{-\infty}^{+\infty} \frac{d \omega_{1}}{\omega_{1}} \int_{-\infty}^{+\infty} \frac{d \omega_{2}}{\omega_{2}} \delta\left(1-\omega_{1}-\omega_{2}\right)
$$

We first have:

$$
\int \mathcal{D} \frac{\omega_{1}}{3}\left(1-2 \omega_{1}\right) \Theta_{1} \partial_{k} \rho_{l 2}=\frac{3}{4 \eta^{2}}-\frac{9}{40}-3 \int_{1 / 2}^{+\infty} d k \frac{1-2 k}{1-k} \beta_{l}(1-k, k) .
$$

Next we have:

$$
\begin{gathered}
\int \mathcal{D} \partial_{k}\left[k^{3}\left(1+\frac{k^{2}}{3}\right) \rho_{l 1} \rho_{l 2}+\frac{2 k}{9}\left(k^{2}\left(1-3 k^{2}\right)+\left(1-4 k^{2}\right) \omega_{1} \omega_{2}-\omega_{1}^{2} \omega_{2}^{2}\right) \rho_{t 1} \rho_{t 2}\right. \\
\left.+\frac{2 \omega_{1}^{2}}{3}\left(1+\frac{\omega_{1}}{k^{2}}-\frac{\omega_{1}^{2}}{k^{2}}\right) \Theta_{1} \rho_{t 2}\right]=-\frac{3}{4}-\frac{40}{27 \pi^{2}} .
\end{gathered}
$$

It is worth mentioning at this stage that all contributions from $k \rightarrow+\infty$ vanish, indeed as it should be since only soft contributions are to contribute. The next piece contains a term the residue part of which we have discussed in the example above. It reads:

$$
\begin{aligned}
& \int \mathcal{D} \frac{\left|\omega_{1}\right|}{3}\left[4 k^{2} \delta_{1} \rho_{l 2}\right.\left.+2 \omega_{1} \delta_{1} \rho_{t 2}+\left(1-2 \omega_{1}\right)\left(1-\frac{\omega_{1}^{2}}{k^{2}}\right) \epsilon\left(\omega_{1}\right) \partial_{k} \Theta_{1} \rho_{t 2}\right] \\
&=3 \int_{1 / 2}^{+\infty} \frac{d k}{1-k}\left(2 k \beta_{l}(1-k, k)+\beta_{t}(1-k, k)\right) .
\end{aligned}
$$


The next pieces are more delicate to handle: they need to work out derivatives of delta functions in the presence of other delta functions with different arguments. Nevertheless that can be done and we get:

$$
\begin{aligned}
& \int \mathcal{D} \frac{1}{6} {\left[2 \omega_{1} k\left(1-\frac{\omega_{1}^{2}}{k^{2}}\right) \partial_{k} \Theta_{1} \partial_{k} \rho_{t 2}+\frac{\omega_{1}}{k}\left(k^{2}-\omega_{1}^{2}\right) \partial_{k}^{2} \Theta_{1} \rho_{t 2}\right.} \\
&\left.-\omega_{1} \partial_{k}^{2}\left(\left(k-\frac{\omega_{1}^{2}}{k}\right) \Theta_{1} \rho_{t 2}\right)\right]=\frac{3}{8}-3 \int_{1 / 2}^{+\infty} \frac{d k}{1-k} \beta_{t}(1-k, k) .
\end{aligned}
$$

The next term is worked out along the same lines as the previous one. We have:

$$
\int \mathcal{D} \frac{\omega_{1} k}{6} \Theta_{1} \partial_{k}^{2} \rho_{l 2}=-\frac{9}{8 \eta^{2}}+\frac{27}{80}+\frac{3}{2} \int_{1 / 2}^{+\infty} \frac{d k}{1-k}\left[\beta_{l}(1-k, k)-\left.k \partial_{k} \beta_{l}(\omega, k)\right|_{\omega=1-k}\right] .
$$

The next term is the most tedious: it necessitates the additional expansion of $\partial_{k} \beta_{t}(\omega, k)$ with $\omega=1-\omega_{t}(k)$ to order $k^{2}$. When this is done and the contributions put together, we get:

$$
\int \mathcal{D} \partial_{k}\left[\frac{k^{2}}{9}\left(1-2 k^{2}-4\left(1-k^{2}\right) \omega_{1}+6 \omega_{1}^{2}-4 \omega_{1}^{3}\right) \rho_{t 1} \partial_{k} \rho_{t 2}\right]=\frac{125}{27 \pi^{2} \eta^{2}}-\frac{1153}{189 \pi^{2}}-\frac{289536}{2187 \pi^{4}}
$$

The last term isn't more difficult. It reads:

$$
\int \mathcal{D}\left[-\frac{2}{3} k^{2} \omega_{1}^{2} \epsilon\left(\omega_{1}\right) \partial_{\omega_{1}^{2}} \delta_{1} \rho_{l 2}\right]=-\frac{3}{2} \int_{1 / 2}^{+\infty} \frac{d k}{1-k}\left(\beta_{l}(1-k, k)+\left.k \partial_{k} \beta_{l}(\omega, k)\right|_{\omega=1-k}\right) .
$$

Putting all these contributions together we obtain:

$$
\begin{aligned}
a_{l 0}^{(2)}= & \left(-\frac{3}{8}+\frac{125}{27 \pi^{2}}\right) \frac{1}{\eta^{2}}-\frac{21}{80}-\frac{1433}{189 \pi^{2}}-\frac{289536}{2187 \pi^{4}} \\
& -3 \int_{1 / 2}^{+\infty} \frac{d k}{1-k}\left[(1-4 k) \beta_{l}(1-k, k)+\left.k \partial_{k} \beta_{l}(\omega, k)\right|_{\omega=1-k}\right]
\end{aligned}
$$

Note that the integral in the above equation (and all the other similar ones actually) is finite. We can evaluate it numerically and we get:

$$
a_{l 0}^{(2)}=\frac{0.09408}{\eta^{2}}-4.45366
$$

Hence, as announced, $a_{l 0}^{(2)}$ is nonzero and quite divergent. Via eq (1), this means $\gamma_{l}(0)$ is itself infrared-divergent. Indeed, using the value $a_{l 0}^{(1)}=6.63538$, we get:

$$
\gamma_{l}(0)=\frac{g^{2} N_{c} T}{24 \pi}\left(\frac{0.09408}{\eta^{2}}+2.18172\right) .
$$


We resume our initial discussion. Remember that the calculation of both $\gamma_{t}(0)$ and $\gamma_{l}(0)$ is performed solely within the framework of the htl-resummation scheme. This latter treats only the two scales $T$ and $g T$ and we have already remarked that the scale $g^{2} T$ may play an important role in QCD at high $T$. This suggests that the scheme as it is may not be robust enough at scales lower than $g T$. One natural way this lack of robustness may manifest itself is via an infrared sensitivity of the scheme when it comes to deal with quantities that to lowest order are already of magnitude $g^{n} T, n \geq 2$. We think the divergence in $\gamma_{l}(0)$ we report in this letter is a direct manifestation of this infrared sensitivity.

One may then ask why the result is finite for $\gamma_{t}(0)$ whereas we find an infrared divergence for $\gamma_{l}(0)$. It is pertinent to note in this respect that there is a main difference between the calculation in the htl scheme of $\gamma_{l}(0)$ and that of $\gamma_{t}(0)$ : in order to get $\gamma_{l}(0)$, we already need an unavoidable expansion to order $p^{2}$ of the imaginary part of the effective self-energy whereas for $\gamma_{t}(0)$ we do not [5,11]. As a matter of fact, we have determined the analytic expression of the transverse-gluon damping rate $\gamma_{t}(p)$ to order $p^{2}$. The coefficient $a_{t 0}$, that of the zeroth order in $p^{2}$, is finite and as we said, equal to $a_{l 0}^{(1)}$ of eq (3) [5]. But a preliminary investigation shows that the coefficient $a_{t 1}$, that of order $p^{2}$, has contributing terms which are divergent in the infrared [14]. It seems then that the expansion of the gluon effective selfenergies * $\Pi$ of the htl scheme in powers of the soft momentum $p$ is infrared sensitive beyond zeroth order. $\gamma_{l}(0)$ necessitating such an expansion to order $p^{2}$, hence an infrared-divergent result.

One may jump to conclude that the htl scheme is robust in the infrared, and that all this simply means that the expansion in powers of the soft momentum of the gluon effective self-energies is not valid in the first place. But remember that this expansion is necessary for $\gamma_{l}(0)$; it is 'part of' its definition, so to speak. Since on physical grounds the damping rates have to be finite for soft momenta, any consistent calculation scheme must allow for a small- $p$ expansion with finite coefficients. If the expansion yields infinite coefficients, it only means that the scheme in which the calculation is performed needs to be cured and/or improved upon, not that the principle of the expansion itself is invalid. It is our opinion that this is the case for the htl scheme in the infrared sector. In other words, an infraredimproved resummation scheme would still allow for the same expansion but would remove the infrared sensitivity of the actual htl scheme.

In fact, the infrared sector is not the first instance in which the htl scheme yields infinities. Indeed, colinear singularities do appear when external light-like momenta are involved and in response to this, the scheme has been subjected to improvement in [13] where an improved action that removes these singularities is proposed. We think the same treatment is necessary in the infrared sector but the situation is more problematic here. Indeed, such an improvement would very likely necessitate the determination of the magnetic mass which is mostly believed to intervene in a nonperturbative way. It may also be that the story does 
not end at the scale $g^{2} T$ and new physics may manifest itself at lower scales $g^{n} T$ with $n>2$, though nothing is suggesting this for the moment. In any case, it is pertinent to note that besides the result we obtain for $\gamma_{l}(0)$, what we think is also interesting in this work is that it sets a calculable framework in which the infrared divergences appear explicitly and by the same token where to test an improved htl scheme when found. In the meantime, we can have a very rough estimate of the scale at which the htl scheme starts to be sensitive. If we demand that $\gamma_{l}(0)$ be equal to $\gamma_{t}(0)$, we may set $a_{l 0}^{(2)}$ equal to zero, which yields $\eta=0.145$ (in units of $m_{g}$ ). However, one should be prudent in considering this value as an estimation, even rough, of the magnetic mass.

Another interesting point to discuss is the logarithmic behavior in $\gamma(p)$ with $p$ soft but nonzero we mentioned in our introductory remarks [10]. More precisely, one argues that $\gamma(p) \sim f(p) \ln \left(m_{\mathrm{mag}} / m_{\text {elec }}\right)$, where $f(p)$ is a well behaved function and the ratio of the magnetic mass to the electric one is (at least) of order $g$. Now as we suggested, our cut-off $\eta$ can be thought of representing the magnetic scale, and thus in units of $m_{\text {elec }}$ (denoted in our work by $m_{g}$ ), it would also be of order $g$ at least. We notice then the absence of $\ln \eta$ in the divergent behavior we obtain for $\gamma_{l}(0)$, see (24). This fact can be used to put back into question the validity of the expansion we make. In particular, one may argue that a term of the form $f(p) \ln p$ should be present in the expansion of the effective self-energies ${ }^{*} \Pi$, a term more in line with the logarithmic behavior. First of all, such a sort of terms is ruled out by the nature of the small-p calculation itself and there is really no subtlety in this as one can straightforwardly get convinced by following the steps reported in [1]. Also, we stress that the htl scheme must allow for the expansion in powers of $p$ and it actually does. What simply happens is that the coefficients of the expansion it yields are infrared sensitive, which means that the scheme is still yet not 'complete' in this infrared sector; in other words, it does not resum diagrams of order $g^{2} T$ and smaller whereas our result suggests there is a need for such a further resummation in one way or another. Once the scheme is properly improved upon, then the new coefficients will cease to diverge.

But the point about the absence of $\ln \eta$ in the expression of $\gamma_{l}(0)$ still remains. Indeed, if the $\ln \eta$ is present in the expression of $\gamma(p)$, then one should expect it to remain present in that of $\gamma(0)$. It is not difficult to understand its absence in the transverse case. Indeed, when we expand $\gamma_{t}(p)$ to order $p^{2}$, the coefficient $a_{t 0}$ is finite but the coefficient $a_{t 1}$ is infrared-divergent, in particular the presence of $\ln \eta$ in it is not ruled out from the outset. Therefore, when $p$ is small but different from zero, the coefficients containing the infrared pieces dominate over the finite ones like $a_{t 0}$. But precisely at $p=0$, only the coefficient $a_{t 0}$ survives and it is finite, hence the absence of $\ln \eta$ or any other infrared-sensitive piece. However, the situation is more delicate in the longitudinal case: this argument cannot be carried forward there since already $a_{l 0}$ is divergent and does not contain a $\ln \eta$ term. Actually the longitudinal situation puts into light a problem we have not raised thus far. Indeed, 
Besides $\ln \eta$, we expect to find power-like divergences (some higher than $1 / \eta^{2}$ ) in $a_{t 1}$. These divergences are more severe than $\ln \eta$ and yet have not been reported in the literature. This issue is well beyond the scope of the present work and we defer its discussion until we finish the disentanglement of all the infrared pieces in $\gamma_{t}(p)$ to order $p^{2}$ [14].

Finally, we have limited ourselves thus far to discussing the gluonic sector only but certainly quarks play an important role too in the structure of hot QCD. They themselves acquire a thermal mass $m_{f}$ to order $g T$ and their damping rates start also at the scale $g^{2} T$, which means they are not a priori shunned from infrared sensitivity.

\section{ACKNOWLEDGMENTS}

We deeply thank Asmaa Abada for all her help and encouragements. O.A. would also like to thank ICTP at Trieste for their kind hospitality where some of the later part of this work was carried. She had the opportunity there to carry constructive discussions with Samina S. Masood. 


\section{REFERENCES}

[1] Very early work was done in the case of QED by V.P. Silin, Zh. Eksp. Teor. Fiz 38 (1960) 1577 [Sov. Phys. JETP 11 (1960) 1136]; V.N. Tsytovich, Zh. Eksp. Teor. Fiz 40 (1961) 1775 [Sov. Phys. JETP 13 (1961) 1249]; O.K. Kalashnikov and V.V. Klimov, Yad. Fiz. 31 (1980) 1357 [Sov. J. Nucl. Phys. 31 (1980) 699]; V.V. Klimov, Yad. Fiz. 33 (1981) 1734 [Sov. J. Nucl. Phys. 33 (1981) 934]; Zh. Eskp. Teor. Fiz 82 (1982) 336 [Sov. Phys. JETP 55 (1982) 199]; H.A. Weldon, Phys. Rev. D26 (1982) 1394.

[2] A representative set of works is D.J. Gross, R.D. Pisarski and L.G. Yaffe, Rev. Mod. Phys. 53 (1981) 43; K. Kajantie and J. Kapusta, Ann. Phys. (N.Y.) 160 (1985) 477; T.H. Hansson and I. Zahed, Phys. Rev. Lett. 58 (1987) 2397; Nucl. Phys. B292 (1987) 725; U. Heinz, K. Kajantie and T. Toimela, Phys. Lett. B183 (1987) 96; Ann. Phys. (N.Y.) 176 (1987) 218; R. Kobes and G. Kunstatter, Phys. Rev. Lett. 61 (1988) 392; U. Kraemmer, M. Kreuzer and A. Rebhan, Ann. Phys. (N.Y.) 201 (1990) 223. A more exhaustive list of works is given in reference 1 of E. Braaten and R.D. Pisarski, Phys. Rev. D42 (1990) R2156.

[3] R.D. Pisarski, Phys. Rev. Lett. 63 (1989) 1129.

[4] E. Braaten and R.D. Pisarski, Nucl. Phys. B337 (1990) 569; Phys. Rev. Lett. 64 (1990) 1338; Nucl. Phys. B339 (1990) 310; J. Frenkel and J.C. Taylor, Nucl. Phys. B334 (1990) 199; reference [3]; Physica A 158 (1989) 246; Nucl. Phys. A498 (1989) 423c. The htl scheme is well discussed now in M. Le Bellac, 'Thermal Field Theory', Cambridge University Press, 1996.

[5] E. Braaten and R.D. Pisarski, Phys. Rev. D42 (1990) R2156.

[6] J.C. Taylor and S.M.H. Wong, Nucl. Phys. B346 (1990) 115; E. Braaten and R.D. Pisarski, Phys. Rev. D45 (1992) R1827.

[7] R. Efraty and V.P. Nair, Phys. Rev. Lett. 68 (1992) 2891.

[8] P.F. Kelly, Q. Liu, C. Lucchesi and C. Manuel, Phys. Rev. Lett. 72 (1994) 3461; Phys. Rev. D 50 (1994) 4209; R. Jackiw, Q. Liu and C. Lucchesi, Phys. Rev. D 49 (1994) 6787.

[9] A.V. Smilga, Phys. Rep. 291 (1997) 1; F. Flechsig, A.K. Rebhan and H. Schulz, Phys. Rev. D52 (1995) 2994; A.K. Rebhan, Nucl. Phys. B430 (1994) 319; Phys. Rev. D48 (1993) R3967.

[10] R.D. Pisarski, Phys. Rev. D47 (1993) 5589; reference [9]; C.P. Burgess and A.L. Marini, Phys. Rev. D45 (1992) R17; A.K. Rebhan, Phys. Rev. D46 (1992) 482; F. Flechsig and 
H. Schulz, Phys. Lett. B349 (1995) 504; H. Schulz, Nucl. Phys. B413 (1994) 353. See also E. Braaten, Phys. Rev. Lett. 74 (1995) 2164. See also a discussion in real-time formalism in S.S. Masood and M.Q. Haseeb, Astroparticle Physics 3 (1995) 405. See in the context of QED and with a different approach J.P. Blaizot and E. Iancu, Phys. Rev. D55 (1997) 973; Phys. Rev. Lett. 76 (1996) 3080. See also in the context of scalar QED M.H. Thoma and C.T. Traxler, Phys. Lett. B378 (1996) 233.

[11] A. Abada, O. Azi and K. Benchallal, Phys. Lett. B425 (1998) 158.

[12] R. D. Pisarski, Physica A 158 (1989) 146; Nucl. Phys. B309 (1988) 476.

[13] F. Flechsig and A.K. Rebhan, Nucl. Phys. B464 (1996) 279; R. Baier, S. Peigné and D. Schiff, Z. Phys. C 62 (1994) 337; P. Aurenche, T. Becherrawy and E. Petitgirard, preprint ENSLAPP-A-452-93.

[14] A. Abada and O. Azi, work in progress. 